The analysis incorporated 16 trials and included 11,906 premenopausal women with early breast cancer who were followed up for a median of 6.8 years. In patients with hormonereceptor-positive tumors, recurrence rates were lower in patients receiving $\mathrm{LHRH}$ agonists alone than in patients receiving no systemic therapy, but this difference was not significant because of the small number of patients included in the comparison. The addition of $\mathrm{LHRH}$ agonists to tamoxifen, chemotherapy, or both, however, resulted in a $12.7 \%$ reduction in recurrence $(P=0.02)$ and a $15.1 \%$ reduction in death after recurrence $(P=0.03)$ in hormone-receptorpositive patients. LHRH agonists had similar effects to chemotherapy in these women, but not in women with hormone-receptor-negative tumors. The addition of LHRH agonists after chemotherapy significantly reduced rates of recurrence, death after recurrence and all deaths in women aged 40 years or younger, but not in older patients. It is possible that use of an LHRH agonist following chemotherapy benefits only women without amenorrhoea after chemotherapy.

Original article LHRH-agonists in Early Breast Cancer Overview group (2007) Use of luteinising-hormone-releasing hormone agonists as adjuvant treatment in premenopausal patients with hormone-receptor-positive breast cancer: a meta-analysis of individual patient data from randomised adjuvant trials. Lancet 369: 1711-1723

\section{Upfront letrozole reduces risk of early breast cancer relapse in high-risk patients}

The optimum role of aromatase inhibitors such as letrozole in the treatment of hormonereceptor-positive breast cancer in postmenopausal women remains uncertain. The ongoing BIG 1-98 trial is evaluating the relative benefits of letrozole and tamoxifen given alone and sequentially. Mauriac et al. have analyzed data from the first 2 years of this trial in order to assess the effects of initial treatment with letrozole versus tamoxifen on the rate of early relapse in estrogen-receptor-positive and/or progesterone-receptor-positive breast cancer.

Of 7,707 eligible patients, 285 (3.7\%) had an early (within 2 years) relapse. Early relapse rates were $3.1 \%$ for patients who had received letrozole, and $4.4 \%$ for those who had received tamoxifen. These results were consistent with an earlier analysis of BIG 1-98 data, which indicated that letrozole increased the likelihood of disease-free survival. Multivariate analysis, adjusted for significant prognostic factors, identified letrozole therapy as an independent predictor of reduced rates of early relapse; differences in early relapse hazards between letrozole and tamoxifen seemed to emerge around 1 year after randomization. When patients were grouped on the basis of prognostic factors, letrozole was significantly better than tamoxifen at preventing early relapse in most subgroups. In particular, those patients at a high risk for early relapse seemed to show a greater than average benefit from letrozole relative to tamoxifen. Patients at intermediate risk might be more suited to sequential therapy - the second primary analysis of BIG 1-98, scheduled for 2008, will address this hypothesis.

Original article Mauriac L et al. (2007) Predictors of early relapse in postmenopausal women with hormone receptorpositive breast cancer in the BIG 1-98 trial. Ann Oncol 18: 859-867

\section{Study finds no evidence of cancer risk from blood transfusion}

The risk of contracting an infectious disease via a blood transfusion is known to be extremely low; however, the risk of chronic disease transmission is less clear. Several studies have examined the possible transmission of cancer from blood donors to recipients, but the results have been conflicting. To further investigate this risk, Edgren et al. conducted a retrospective cohort study using data gathered by all computerized blood bank registers in Sweden (1968-2002) and Denmark (1984-2002). Linkages with nationwide health-care registries enabled the researchers to identify 354,094 transfusion recipients for whom complete data were available.

Over a follow-up of 3,200,800 person-years, 29,651 primary cancers occurred in transfusion recipients. Blood from donors who developed cancer within 5 years of donation was given to 12,012 individuals, 978 of whom subsequently developed cancer. Regression analyses of cancer incidence adjusted for sex, age, and number of transfusions found no increased risk of cancer in those who had been exposed to precancerous blood products compared with those who had received blood from a noncancerous donor (relative risk 1.00, 95\% $\mathrm{Cl} 0.94-1.07$ ). 